\title{
ПУТИ ПРАКТИЧЕСКОЙ РЕАЛИЗАЦИИ концепции туманных вычислений
}

\author{
А.Пинчук, директор ООО "НТЦ ПРОТЕЙ", \\ Н.Соколов, д.Т.н., директор по науке ООО "ПРОТЕЙ СпецТехника", \\ В.Фрейнкман, директор по маркетингу и системным исследованиям ООО "НТЦ ПРОТЕЙ"
}

УДК 621.391, DOI: 10.22184/2070-8963.2019.78.1.56.61

Обсуждаются возможные пути практической реализации концепции туманных вычислений с учетом существующего уровня развития телекоммуникационной системы страны. Предлагается разработать макет, включающий аппаратно-программные средства, которые позволят сформулировать ряд важных принципов применения туманных вычислений. Акцентируется внимание на важности использования отечественных разработок с целью минимизации риска, обусловленного проблемами информационной безопасности.

\section{ЧЕТЫРЕ ВОПРОСА, ТРИ АЛЬТЕРНАТИВЫ}

Ряд проблем, касающихся практической реализации туманных вычислений, изложен в предыдущих публикациях [1, 2]. В этой статье, завершающей цикл работ по туманным вычислениям, рассматриваются четыре вопроса:

- разработка макета телекоммуникационного оборудования, которое необходимо для проведения вычислений в "тумане";

- тестирование новых аппаратно-программных средств, создаваемых для воплощения идей рассматриваемой концепции;

- обеспечение информационной безопасности за счет использования преимущественно отечественных разработок;

- постановка новых научно-технических задач, требующих проведения дополнительных исследований.

Следует подчеркнуть, что пути практической реализации туманных вычислений рассматриваются с точки зрения задач, возникающих перед телекоммуникационной системой. При этом решения, касающиеся информационной системы, учитываются только в виде требований к производительности устройств коммутации и пропускной способности транспортных ресурсов. Уместно также акцентировать внимание читателей на том факте, что слово "вычисление" применительно к проведению соответствующих операций в "тумане" не обязательно связано с математическими преобразованиями. В данном случае разумной представляется трактовка, принятая в теории информации: вычисление - это получение из входных данных нового знания [3].

Путь реализации концепции любого рода можно рассматривать как одну из трех альтернатив:

- приобретение импортного оборудования, которое далее будет использоваться в качестве "черного ящика" [4] со всеми вытекающими последствиями;

- разработка полностью отечественного комплекса всех аппаратно-программных средств;

- компромиссное решение, в котором минимизируются риски информационной безопасности за счет применения отечественных разработок в критически важных компонентах используемых аппаратно-программных средств.

Взвесив все pro et contra, следует выбрать третью альтернативу. Российские производители телекоммуникационного оборудования доказали способность качественно создавать инновационные продукты 


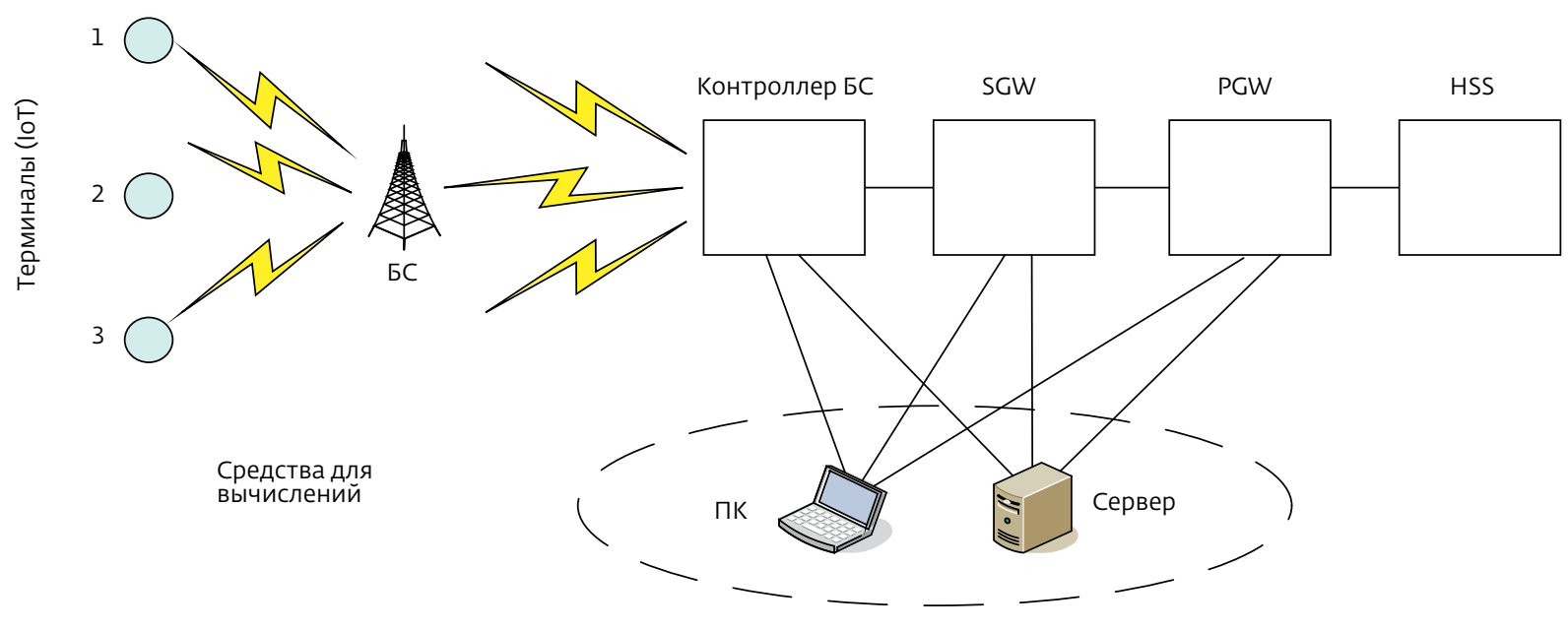

IoT (Internet of Things) - Интернет вещей, БС - базовая станция, Пк - персональный компьютер

SGW (Serving Gateway) - обслуживающий шлюз, PGW (Packet data network Gateway) - шлюз для сетевой пакетной передачи данных, HSS (Home Subscriber Server) - сервер абонентских данных.

Рис.1. Структура предлагаемого макета туманных вычислений

$[5,6]$. Именно такой путь разработки макета телекоммуникационных средств, предназначенных для проведения туманных вычислений, рассматривается в данной статье.

\section{РАЗРАБОТKA MAKETA}

\section{ТЕЛЕКОММУНИКАЦИОННЫХ СРЕДСТВ ТУМАННЫХ ВЫЧИСЛЕНИЙ}

Состав технического задания (Т3) на макет, позволяющий проверить ряд основных предлагаемых решений для реализации концепции туманных вычислений, определяется верхней частью модели, которая была приведена в статье [1] на рис.3. В этом фрагменте модели были представлены функциональные модули туманных вычислений, выполняющие ключевые операции по обмену данными и их обработке. Структура предлагаемого макета туманных вычислений показана на рис.1 как часть ранее рассмотренной модели. Ключевые аппаратно-программные средства SGW, PGW и HSS, выполняющие основные функции, возложенные на телекоммуникационную систему, предполагается реализовать в полном объеме. Средства обработки информации, которые в составе макета выполняют функции туманных вычислений, уместно ограничить двумя типами - ПК и сервер.

Основным разделом Т3 на макет туманных вычислений следует считать "Технические требования", так как именно они определяют функциональные возможности предлагаемой разработки. При этом технические требования на ряд компонентов макета уже известны, но для аппаратно-программных средств SGW, PGW и HSS их необходимо разработать.

Технические требования к аппаратно-программным средствам, предназначенным для создания системы туманных вычислений, определяются основными функциональными задачами, которые возлагаются на блоки SGW, PGW и HSS. Кроме того, для этих блоков следует определить атрибуты, которые зависят от величины обслуживаемого трафика: пропускную способность (throughput), производительность (packet processing и call transaction rates), требования к ресурсам памяти (significant memory resources), а также ряд других показателей.

Следует отметить, что радиооборудование в составе макета может быть импортным, но только до появления полноценных российских аналогов. Данное утверждение относится к терминалам ІоТ. После проведения испытаний макета целесообразно предпринять все необходимые меры, чтобы концепция туманных вычислений начала воплощаться на практике на базе отечественного оборудования.

Важными задачами, решаемыми при помощи макета, следует считать выбор принципов принятия альтернативных решений по проведению вычислений или в тумане, или в облаке; разработку алгоритмов управления функциональными блоками при 
перегрузках либо отказах отдельных аппаратно-программных средств, а также проверку системы информационной безопасности. С учетом перечисленных выше обстоятельств после формирования технических требований к аппаратно-программным средствам, предназначенным для создания системы туманных вычислений, должны быть разработаны программы и методики для проведения соответствующих испытаний.

При формировании технических требований для блоков SGW, PGW и HSS уместно использовать методологический подход, основанный на трех положениях:

- составление функциональных требований к перечисленным выше блокам с учетом известных стан ${ }^{-}$ дартов, а также ожидаемых изменений и дополнений;

- выбор требований с учетом характеристик пропускной способности, производительности и ресурсов памяти, которые представляются целесообразными для макета туманных вычислений;

- определение набора информационных технологий (например, Big Data, Data Mining и им подобных), применение которых позволит доказать эффективность туманных вычислений с точки зрения потенциальных пользователей.

Пример подобного методологического подхода иллюстрирует рис.2. Он выполнен в виде куба, грани которого содержат информацию по каждому из трех сформулированных выше положений. Надписи на трех гранях куба содержат краткие формулировки тех положений, которые составляют полноценные технические требования к аппаратно-программным средствам, которые необходимо разработать для реализации технологии туманных вычислений. Составление детальных технических требований - предмет опытно-конструкторской работы.

\section{ЗАДАЧИ ТЕСТИРОВАНИЯ АППАРАТНО- ПРОГРАММНЫХ СРЕДСТВ}

С методологической точки зрения задачи тестирования аппаратно-программных средств, предназначенных для туманных вычислений, аналогичны проблемам исследования "черного ящика" [4]. Процессы тестирования интерфейсов и протоколов разработаны для аналогичных технологий и вряд ли потребуют существенного развития. Это утверждение справедливо и для тестирования функционала, присущего технологии туманных вычислений.

С точки зрения тестирования наиболее неопределенными атрибутами туманных вычислений станут, по всей видимости, качество обслуживания, надежность и информационная безопасность. Для качества обслуживания неопределенность заключается, в первую очередь, в плохой предсказуемости колебаний трафика. С этой точки зрения полезен подход к тестированию, основанный на модели ТьюкиХьюбера [7], которая позволяет учесть весьма существенные изменения интенсивности трафика в течение короткого промежутка времени. При тестировании надежности следует провести и анализ живучести [8] системы туманных вычислений за счет искусственного изъятия из состава макета ряда узлов туманных вычислений и вычислительных средств, расположенных в облаке. Кроме того, желательно проверить влияние "времени жизни" используемых автономных источников электропитания (ИЭ) для топологий с концентратором и без него, а также возможность обеспечения необходимой аварийной сигнализации при снижении уровня заряда ИЭ ниже критического в самом устройстве или в концентраторе.

Анализ обеспечения заданных требований по информационной безопасности подразумевает тестирование макета при разных моделях угроз, свойственных туманным вычислениям.

Также представляется целесообразным провести тестирование влияния приложений, осуществляющих туманные вычисления, на функционирование основных систем, на чьих ресурсах эти вычисления производятся, и разработать рекомендации по минимизации такого рода влияний.

Для проведения тестирования системы туманных вычислений должны быть разработаны соответствующие программы и методики, учитывающие тот факт, что требования к туманным вычислениям будут меняться в весьма широких пределах. Следовательно, программы и методики изначально должны содержать возможность их адаптации к меняющимся внутренним и внешним условиям использования технологии туманных вычислений.

\section{АСПЕКТЫ ИНФОРМАЦИОННОЙ БЕЗОПАСНОСТИ}

Актуальным задачам обеспечения безопасности (как правило, внимание акцентируется на информационной безопасности) посвящен обширный ряд публикаций в отечественных и зарубежных источниках. В этом разделе статьи предлагается модель, позволяющая проиллюстрировать преимущества использования отечественных решений для дальнейшего развития телекоммуникационных сетей. Эта модель показана на рис.3. Первый путь подразумевает модернизацию сети на основе отечественных разработок, которые не содержат недокументированных возможностей (НДВ). Применение зарубежных аппаратнопрограммных средств (хотя бы частично) допускается в случае выбора второго пути. 
Предполагается, что развитие телекоммуникационной сети предусматривает ее переход из некого состояния "А" в состояние "Б", которое характеризуется более высоким уровнем качества предоставляемых услуг и расширением функциональных возможностей в части технической эксплуатации. В случае выбора первого пути основные проблемы функционирования телекоммуникационной сети будут обусловлены непреднамеренными ошибками в аппаратных и в программных средствах. Эти ошибки неизбежны, но их количество можно минимизировать за счет тщательного анализа допущенных ранее просчетов, а также в процессе тестирования. При выборе второго пути к уже упомянутым выше ошибкам следует добавить как минимум три вида угроз, перечисленных в нижней части рассматриваемой модели.

Момент $\mathrm{T}_{0}$ соответствует началу отрезка времени, до наступления которого обнаружена только часть угроз, влияющих на безопасность телекоммуникационной сети. В дальнейшем могут быть выявлены дополнительные угрозы, которые небыли обнаружены к моменту $\mathrm{T}_{0}$. Следует подчеркнуть, что могут также существовать и плохо прогнозируемые угрозы, которые не только не обнаружены, но и не предсказуемы.

Если выбирать аналогию для сравнения двух путей развития телекоммуникационной сети, то вполне

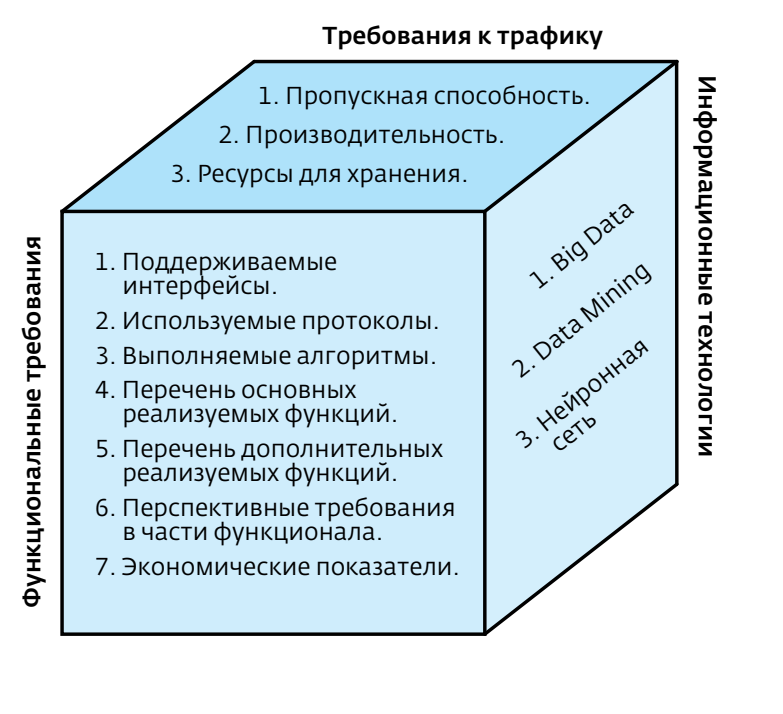

Рис.2. Методологический подход к разработке требований к макету

уместно оперировать сценариями перехода улицы с интенсивным автомобильным движением. Первый сценарий похож на ожидание зеленого сигнала светофора. Риск попасть в дорожно-транспортное 


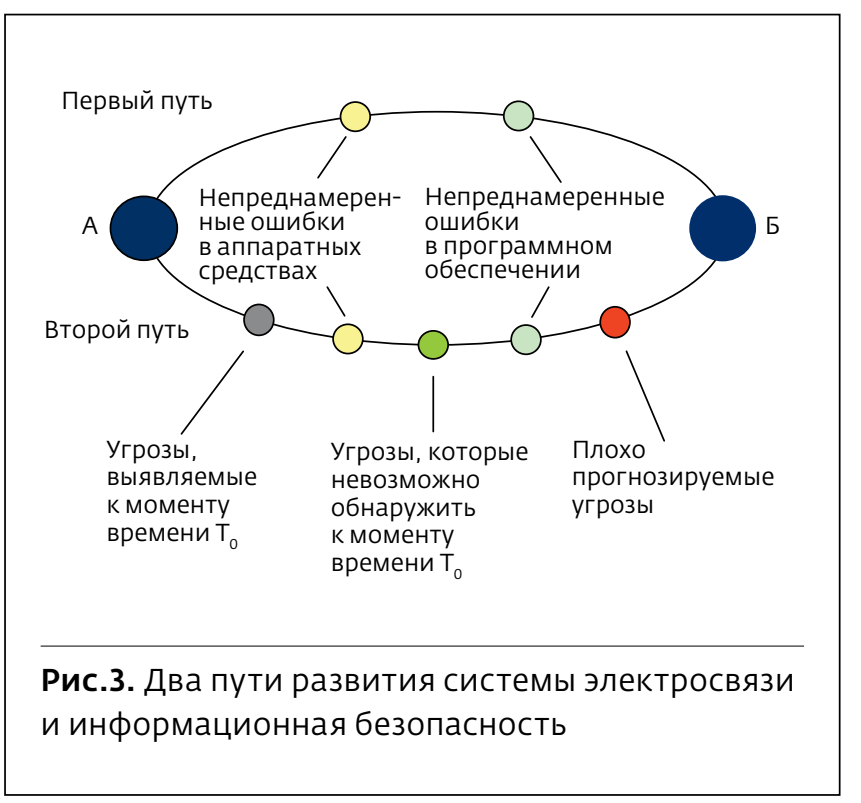

происшествие минимален, но все же он существует из-за возможного отказа тормозов у автомобиля (аналог непреднамеренной ошибки в аппаратных средствах) или сбоя в работе светофора (аналог непреднамеренной ошибки в программном обеспечении). Второй сценарий подобен пересечению улицы без ожидания разрешающего сигнала светофора в расчете на возможность проскочить между всеми движущимися транспортными средствами. Тогда к уже упомянутым рискам следует добавить как минимум два дополнительных фактора. Во-первых, к моменту $\mathrm{T}_{0}$, когда начат переход, существуют необнаруженные визуально угрозы. Во-вторых, можно столкнуться с теми угрозами, которые плохо прогнозируются в силу объективных и субъективных причин.

Необходимо упомянуть еще один аспект применения отечественного и зарубежного оборудования, который касается функциональных возможностей сети и перечня поддерживаемых услуг. По этим показателям в некоторых случаях импортное оборудование имеет ряд не всегда существенных преимуществ. Тем не менее, неизбежно возникает вопрос: стоит ли пожертвовать безопасностью ради преимуществ, которые иногда представляются эффективными, но обычно малоэффективны? Ответ на этот вопрос очевиден операторам сетей электросвязи специального назначения, но пока остается открытым для других эксплуатационных компаний.

Соображения, изложенные в этом разделе, справедливы не только для туманных вычислений. Правда, для туманных вычислений они становятся более актуальными, так как одна из важных сфер приложений - Интернет вещей - привносит дополнительные риски в части комплексной безопасности $[9,10]$, включая, конечно, и ее информационный компонент.

\section{ОБСУЖДЕНИЕ РЕЗУЛЬТАТОВ ИССЛЕДОВАНИЯ}

Идея туманных вычислений определяет актуальное направление для развития не только инфокоммуникационной системы, но и совокупности важных сфер экономической и социальной жизни общества. С этой точки зрения изучение туманных вычислений и определение оптимального пути практической реализации соответствующей концепции представляет собой предмет междисциплинарных исследований и совместных разработок силами специалистов из разных отраслей. С другой стороны, следует акцентировать внимание на том факте, что в настоящее время нет четко сформулированных положений, однозначно определяющих принципы построения всей системы туманных вычислений (в отличие от ряда предшествующих концепций - общеканальной сигнализации, интеллектуальной сети и др. Это означает, что некоторая неопределенность в отношении концепции туманных вычислений сохранится и в обозримой перспективе.

Данное положение - отчасти - не уникально и связано с проблемой сингулярности [11], выражаемой в том числе постоянным ускорением процессов появления новых идей и технологий. Один из путей минимизации возникающих рисков заключается в использовании сценарного подхода, который учитывает возможность существенных изменений каждой концепции в течение сравнительно короткого промежутка времени.

Тем не менее, концептуальные положения туманных вычислений, изложенные в в этой статье и в предшествующих публикациях $[1,2]$, позволяют перейти к практической реализации этой технологии. Движущей силой для распространения туманных вычислений можно считать тандем, состоящий из концепций Интернета вещей и сети 5G. Для реализации обеих концепций в России существует достаточный научно-технический и производственный потенциал, способный стать локомотивом для всей национальной экономики, учитывая уже начавшуюся четвертую промышленную революцию, известную также по названия м "Индустрия 4.0" и "Цифровая экономика".

Ряд проблем, прямо или косвенно связанных с туманными вычислениями, требует проведения дополнительных исследований. К ним относятся: оценка необходимых вычислительных и телекомму никационных ресурсов с учетом специфики объектов применения и прогнозов на обозримую перспективу; нормирование показателей качества обслуживания 
мультисервисного трафика (предоставления инфокоммуникационных услуг); составление детальных технических требований к аппаратно-программным средствам, предназначенным для туманных вычислений; разработка решений по повышению уровня информационной безопасности для различных приложений. К этим задачам следует добавить и разработку отечественной терминологии [13] для туманных вычислений и схожих концепций.

\section{ЛИТЕРАТУРА}

1. Пинчук А.В., Соколов Н.А., Фрейнкман В.А. Общие принципы туманных вычислений // ПЕРВАЯ МИЛЯ. 2018. № 3. С. 38-45.

2. Пинчук А.В., Соколов Н.А., Фрейнкман В.А. Сценарии создания и развития концепции туманных вычислений // ПЕРВАЯ МИЛЯ. 2018. № 4. С. 24-31.

3. Электронный ресурс https://ru.wikipedia.org/wiki/ вычисление.

4. Бейзер Б. Тестирование черного ящика. Технологии функционального тестирования программного обеспечения и систем. - СПб.: Питер, 2004. 318 с.

5. Пинчук А.В., Соколов Н.А. Опыт формирования инновационных решений при разработке телекоммуникационного оборудования // Вестник свя3и. 2017. № 2. С. 3-8.
6. Гольдштейн Б.С. Отечественные производители телекоммуникационного оборудования // Connect! Мир связи. 2008. № 3. С. 2-6.

7. Маршак М.А., Соколов Н.А. Об одной актуальной задаче обеспечения качества обслуживания трафика в NGN // Вестник связи. 2010. № 1. С. 10-12.

8. Попков В.К. Математические модели связности. - Новосибирск: Издательство ИВМиМГ СО PAH, 2006. 490 c.

9. Пинчук А.В., Секереш В.В., Соколов Н.А. Методологический подход к построению системы комплексной безопасности. Часть I // ПЕРВАЯ МИЛЯ. 2015. № 5. C. 58-64.

10. Пинчук А.В., Секереш В.В., Соколов Н.А. Методологический подход к построению системы комплексной безопасности. Часть II // ПЕРВАЯ МИЛЯ. 2015. № 6. С. 52-57.

11. Панов А.Д. Сингулярная точка истории // Общественные науки и современность. 2005. № 1. C. 122-137.

12. Ustundag A., Cevikcan E. Industry 4.0: Managing The Digital Transformation. - Международное издательство Springer. 2018. 293 p.

13. Соколов Н.А. Изменение терминологии в отрасли "Электросвязь" // ПЕРВАЯ МИЛЯ. 2015. № 7. С. 12-15. 